\title{
Review of "Tailored interventions to assist smokers to stop smoking using e- cigarettes (TASSE): Study protocol"
}

Clive Bates

The aims of the study are admirable and its design looks solid, and I look forward to reviewing the final paper. In anticipation of that, I have two questions about the design.

The first question relates to randomisation. In the design, "smokers will be randomly allocated to one of the 32 conditions, which will be permutations of the 5 components". But in reality users have non-uniform non-random preferences for the kind of intervention they are prepared to engage with. The authors note that behavioural support via smoking cessation services has reasonable efficacy, but in practice few people are willing to endure the time commitment and intensity of the intervention. This highlights a feature of behavioural interventions: their impact is the product of their effect on users' behaviour and the number of users willing to engage with the intervention. An effective intervention that few people want to adopt is of little value. Also, different people may have different preferences for engaging with the five support methods. Why randomise them to interventions that they would not choose if given the choice? If interventions that people choose prove to be more more effective, then randomisation will squeeze out possible benefits of user agency. Perhaps the users could be asked which of a five interventions or 32 pairs they would prefer to be allocated to? If that would mean some pairs would no longer have meaning ful statistical power, then maybe they should be dropped and users reassigned to their next preference. Or preference could just be measured before randomisation. If the subjects' preferences clustered in a few pairs, that would be a valuable reality-based finding.

The second question relates to the participants. Why restrict the participation to "smokers who are interested in stopping smoking"? Many vapers report they became 'accidental quitters' after trying e-cigarettes. These would be people who do not define themselves as wanting to quit smoking and possibly think of vaping as "smoking, just differently". One of the interesting characteristics of e-cigarettes is the possible evolution of motivation as use progresses from initial experimentation to a deepening 
commitment. Why select for people declaring a motivation to quit smoking? What about "people who are interested in trying e-cigarettes as an alternative to smoking"?

Neither of these questions are intended as a criticism of the design. They are asking whether there is an opportunity to refine the design to be more closely linked to how vaping works as an alternative to smoking in practice, reflecting the role played by user autonomy, preferences and motivation in behaviour change. I accept that this may be asking to test too many different things in one trial, but I would be grateful if the authors would consider these comments. 